\title{
Delayed Sleep Phase Type Circadian Rhythm Sleep Disorder
}

National Cancer Institute

\section{Source}

National Cancer Institute. Delayed Sleep Phase Type Circadian Rhythm Sleep Disorder.

NCl Thesaurus. Code C95073.

A subtype of circadian rhythm sleep disorder in which the individual exhibits a persistent pattern of late sleep onset and late awakening, which results from an endogenous sleepwake cycle that is delayed relative to the societal norm. 